\title{
Validez de contenido de la escala de barreras para la rehabilitación cardiaca
}

\author{
Content validity of the scale of barriers to cardiac rehabilitation
}

\author{
Juan Carlos Sánchez-Delgado', Adriana Jácome-Hortúa², Sandra Pinzón ${ }^{3}$, Adriana Angarita-Fonseca ${ }^{4}$
}

1 Fisioterapeuta, MSc Actividad Física y Deporte, Docente Universidad de Santander (UDES) y Universidad Santo Tomás. Bucaramanga, Colombia.e-mail: juancarlossanchezd@gmail.com

2 Fisioterapeuta, Esp. Rehabilitación Cardiopulmonar, Docente Universidad de Santander (UDES). Bucaramanga, Colombia. e-mail: AD.JACOME@mail.udes.edu.co

3 Fisioterapeuta, Esp. Entrenamiento Deportivo, Docente Universidad de Santander (UDES). Bucaramanga, Colombia. e-mail: shana7912@hotmail.com

4 Fisioterapeuta, MSc Epidemiología, Docente Universidad de Santander (UDES) y Universidad Santo Tomás. Bucaramanga, Colombia. e-mail: adriangarita@udes.edu.co

Fecha de recepción: Noviembre 22 - 2014

Fecha de aceptación: Septiembre 18 - 2015

Sánchez-Delgado JC, Jácome-Hortúa A, Pinzón S, Angarita-Fonseca A. Validez de contenido de la escala de barreras para la rehabilitación cardiaca. Univ. Salud.2015;17(2): 170 - 176. DOI: http://dx.doi.org/10.22267/rus.151702.2

\section{Resumen}

Introducción: La ausencia de instrumentos con adecuadas propiedades psicométricas adaptados para Colombia, que evalúen las barreras de acceso a los programas de rehabilitación cardiovascular justifica esta investigación. Objetivo: Determinar la validez de contenido de la Escala de Barreras para la Rehabilitación Cardiaca en población colombiana. Métodos y materiales: Se realizó un estudio de pruebas diagnósticas. En la primera fase se requiririeron dos traductores de lengua materna español y uno de lengua materna inglesa para la traducción y retrotraducción del cuestionario original. En la segunda fase se realizó la adaptación transcultural y se evaluó la validez de contenido mediante un panel de cuatro expertos con experiencia en investigación, medicina deportiva y rehabilitación cardiaca. Resultados: El instrumento fue traducido y adaptado al español colombiano. El panel de expertos decidió eliminar el ítem 18 de la escala original. El índice de validez de contenido fue aceptable para la mayoría de los ítems, excepto para los ítems 10,15 y 18, por lo cual se realizaron modificaciones en palabras, frases o conjugaciones verbales según las recomendaciones del panel. Por otra parte, el Indice de Validez de Contenido en relevancia fue de 0,86 y en pertinencia de 0,88. Conclusión: Se cuenta con un instrumento que evalúa barreras de acceso a los programas de rehabilitación cardiovascular adaptada a población colombiana y con validez de contenido, por lo cual los resultados que se obtengan de la aplicación de la escala serán válidos. No obstante, se sugiere continuar con la evaluación de la reproducibilidad del instrumento.

Palabras clave: Validez de las pruebas, enfermedades cardiovasculares, enfermedad coronaria, rehabilitación, cuestionarios. (Fuente: DeCS, Birme).

\begin{abstract}
Introduction: This research was based on the absence of instruments with appropriate psychometric properties, adapted for Colombia, which evaluate the barriers of access to cardiovascular rehabilitation programs. Objective: To determine the validity of content of the barriers scale for the cardiac rehabilitation in Colombian population. Methods and materials: A study of diagnostic tests was done. In the first phase, two native speakers of Spanish and one of English were required to translate and retro translate the original questionnaire. In the second phase, the transcultural adaptation was done and the validity of content was assessed by a panel of four experts with experience in research, sports medicine and cardiac rehabilitation. Results: The instrument was translated and adapted to the Colombian Spanish. The panel of experts decided to remove the item 18 from the original scale. The content validity index was acceptable for the majority of the items, except for items 10, 15 and 18, where modifications were made in terms of words, phrases or conjugations according to the
\end{abstract}


recommendations of the panel. On the other hand, the IVC in relevance was 0.86 and relevance of 0.88 . Conclusion: There is an instrument that evaluates the barriers of access to cardiovascular rehabilitation adapted to the Colombian population programs and with content validity, so the results to be obtained from the application of the scale will be valid. However, it is suggested to continue with the evaluation of the reproducibility of the instrument.

Keywords: Validity of testing, cardiovascular diseases, coronary heart disease, rehabilitation, questionnaires. (Source: DeCS, Bireme).

\section{Introducción}

Las enfermedades cardiovasculares son la primera causa de muerte en el mundo, a pesar del extenso conocimiento de las formas de prevención y tratamiento farmacológico, quirúrgico y de estilos de vida. La rehabilitación cardiaca (RC), hace parte de las herramientas utilizadas para tratar este tipo de patologías, y uno de sus efectos más importantes es la reducción de nuevos eventos adversos y de la mortalidad en un $25 \%_{{ }_{1-3}}$

No obstante, es importante resaltar que entre el 7,5\% al $25 \%$ de la población apta para participar en un programa de RC lo hace, y de estos, el 50\% desertan prematuramente. $_{4-9}$ Según Mclark et al., existen barreras personales y contextuales que limitan dicha participación, las primeras incluyen las apreciaciones acerca de las nociones negativas de salud y las reacciones de enfrentar una enfermedad cardiaca, entre las contextuales están la dificultad para acceder a transporte y la distancia entre el hogar y el sitio donde se lleva a cabo el programa. Adicionalmente, se consideran otras barreras como pertenecer al sexo femenino, ser adulto mayor, presencia de comorbilidades, estar desempleado, pertenecer a un estrato socioeconómico bajo y como el más fuerte predictor de participación en un programa de RC está la prescripción médica. ${ }_{10}$

No son muchos los instrumentos que evalúan las barreras para la participación en los programas de RC, entre los encontrados está el creado por Fernández et al., que evalúa los obstáculos para acceder a los servicios de RC, mostrando una adecuada consistencia interna (alpha de Cronbach = 0,89), no obstante su validación fue realizada con pacientes australianos sometidos a angioplastia coronaria, por lo cual su utilidad en otros grupos es desconocida. ${ }_{11}$
El instrumento utilizado en este estudio "Escalas de Barreras para la Rehabilitación Cardiaca" (EBRC) es de origen canadiense, tipo Likert de 5 puntos y fue creado con 22 items, que evalúan cuanto pueden impedir la asistencia a un programa de RC los factores personales, logísticos, los conflictos de trabajo y tiempo, la morbilidad y el estado funcional en cualquier sujeto con enfermedad cardiovascular. Además, presenta una confiabilidad aceptable en la versión inglés (alpha Cronbach de 0.88, $\mathrm{CCI}=0.64)$ y portugués (alpha Cronbach de 0.88, $\mathrm{CCI}=0,68)_{{ }_{4,12,14}}$ Aunque existe una versión en español del instrumento, reconocida por los autores, no se le ha realizado ningún proceso de validación. ${ }_{15}$

Al utilizar una escala foránea que define un constructo como el de "barreras", se requiere un proceso de adaptación cultural y la posterior validación del instrumento. $_{16}$ En relación con la validez, existen varios tipos como la facial, de constructo, de criterio y de contenido; esta última fue la evaluada en el presente estudio y es definida por Hernández et al., como "grado en que un instrumento refleja un dominio especifico de contenido de lo que se mide" ${ }_{17}$ Por lo anterior y ante la ausencia de escalas locales que permitan medir las barreras para participar en un Programa de Rehabilitación Cardiaca (PRC), el objetivo de esta investigación fue determinar la validez de contenido de la EBRC.

\section{Materiales y métodos}

Se realizó un estudio de evaluación de tecnologías diagnósticas, donde se determinó la validez de contenido de la EBRC. La unidad de análisis fue la evaluación realizada por 4 expertos, 2 fisioterapeutas especialistas en rehabilitación cardiaca, un médico epidemiólogo y un deportólogo. Cada experto evaluó 
la relevancia (importancia de cada ítem para la evaluación de barreras para la rehabilitación cardiaca en población colombiana) y la pertinencia (se refiere a que los ítems evalúan lo que pretenden evaluar) de los 22 ítems de la escala. De este modo se generaron las variables del estudio: 22 de relevancia y 22 de pertinencia.

El estudio se desarrolló en tres fases, que inician con la solicitud para el uso a la autora de la escala "Sherry Grace", posteriormente se realiza el proceso de adaptación transcultural teniendo en cuenta los pasos metodológicos descritos por García et al., ${ }_{18}$ el cual incluye la validación lingüística, caracterizada por la traducción de la escala del inglés al español, proceso desarrollado por un profesional de la salud bilingüe de lengua materna castellano, seguida por la retro-traducción de la versión 1 al inglés, proceso realizado por un profesional de la salud bilingüe de lengua materna inglesa. Finalmente, las versiones se sometieron al panel de expertos, los cuales emitieron su juicio sobre cada uno de los ítems y la escala en general.

Para evaluar la validez de contenido se elaboró un instructivo y plantilla de registro donde se puede describir la pertinencia y relevancia de cada ítem, utilizando una escala de 2 puntos, que va desde: $0=$ no pertinente o no relevante y $1=$ pertinente o relevante según sea el caso. Adicionalmente, se dejó un espacio en donde el experto registró sus observaciones de cada ítem y del cuestionario en general.

\section{Análisis estadístico}

Una vez diligenciadas las plantillas, los datos se analizaron usando Excel versión 2007. El análisis estadístico incluyó el cálculo del índice de relevancia (IR) y pertinencia (IP) de cada ítem, que se obtuvo de dividir el número de expertos que calificaron en 1 la pertinencia y/o la relevancia del ítem, por el total de expertos que evaluó el ítem. Se consideró un ítem adecuado o pertinente si el IR o IP fue $\geq 0.8 ._{18} \mathrm{El}$ Índice de validez de contenido (IVC) en relevancia y en pertinencia para toda la escala se calculó teniendo en cuenta el promedio de los IR e IP de los 22 ítems; se consideró que la escala fue relevante y pertinentes si los IR e IP para toda la escala fue de $\geq 0.80 ._{19}$

\section{Consideraciones éticas}

El estudio fue aprobado por el Comité de Ética de la Universidad de Santander, y se clasifica como una investigación sin riesgo, en el marco del artículo 11 de la Resolución 8430 de 1993 para investigación en seres humanos, ya que no se realiza ninguna intervención de las variables del estudio.

\section{Confidencialidad}

Cada uno de los expertos firmó un consentimiento, donde se comprometen a no revelar la información, ni utilizarla para otros propósitos fuera del de cumplir con los objetivos de este estudio.

\section{Resultados}

En la tabla 1 se describe los índices de validez de contenido de los ítems respecto a su relevancia y pertinencia. Los únicos que no presentan resultados aceptables son: "Por motivos de viajes (por ejemplo, negocios, situaciones y/o, paseos familiares)"; "Yo me siento viejo" y "yo mismo puedo manejar mi problema del corazón y no necesito ayuda", con un IVC para la relevancia y pertinencia de $(0,5)$. Adicionalmente se observa el índice de validez de contenido de la escala (IVC) aceptable para la pertinencia $(0,88)$ y relevancia $(0,86)$.

De los ítems con IR o RP no aceptables, un experto sugiere eliminar el que hace referencia a "yo me siento viejo", porque expresa que se puede cubrir con el ítem "yo encuentro el ejercicio fatigante y/o doloroso". Así mismo, sugiere eliminar el ítem "por motivos de viajes (por ejemplo, negocios, situaciones y/o, paseos familiares)" el cual está implícito en el ítem "por falta de tiempo (por ejemplo: yo estoy muy ocupado, y/o tengo inconvenientes con los horarios de las sesiones)". Otras observaciones planteadas por los expertos y tenidas en cuenta fueron: del ítem 2 “manutención no es una palabra común" y del ítem 4 "actividades de limpieza no es un término habitual", sugiere cambiarlo por "actividades del hogar".

Entre las observaciones generales emitidas por los expertos encontramos: "Algunos conceptos podrían no ser aplicables por su condición de género y edad"; 
"debe adaptase al nivel socioeconómico"; "ampliar ejemplos para incluir situaciones que puedan ocurrir a personas de diferentes estratos sociales". Teniendo en cuenta todo lo anterior, se creó un documento final con su respectiva traducción, con el objetivo de mejorar los niveles de aceptabilidad de cada uno de los ítems y de la escala en general.

\section{Tabla 1. Relevancia y pertinencia de los Ítems Escala de Barreras para la Rehabilitación Cardiaca EBRC}

\begin{tabular}{|c|c|c|c|}
\hline $\mathbf{N}^{\circ}$ & Ítem & IR & IP \\
\hline 1 & $\begin{array}{l}\text {...La distancia (por ejemplo, no se encuentra ubicado en su barrio donde vive, le queda muy lejos para su } \\
\text { desplazamiento) }\end{array}$ & 0,8 & 1,0 \\
\hline 2 & $\begin{array}{l}\text {...Al costo (por ejemplo, el pagar parqueadero y gasolina, transporte público, gastos de manutención en la } \\
\text { ciudad) }\end{array}$ & 1,0 & 1,0 \\
\hline 3 & $\begin{array}{l}\text {...A problemas con el transporte (por ejemplo, no pasa la ruta, tengo dificultad para el acceso al sistema de } \\
\text { transporte) }\end{array}$ & 1,0 & 1,0 \\
\hline 4 & $\begin{array}{l}\text {...A responsabilidades familiares (por ejemplo, el cuidado de sus hijos, nietos, parientes, actividades de } \\
\text { limpieza) }\end{array}$ & 1,0 & 1,0 \\
\hline 5 & ...Yo no sabía acerca de la rehabilitación cardiaca (por ejemplo, el médico no dijo sobre esto) & 1,0 & 1,0 \\
\hline 6 & $\begin{array}{l}\text {...Yo no necesito rehabilitación cardiaca (por ejemplo, yo me siento bien, mi problema cardíaco está } \\
\text { controlado, no es grave) }\end{array}$ & 1,0 & 1,0 \\
\hline 7 & ...Yo Hago actualmente ejercicio en mi casa o en mi comunidad & 0.8 & 0.8 \\
\hline 8 & ... Por el clima & 0.8 & 0.8 \\
\hline 9 & ...Yo encuentro el ejercicio fatigante y/o doloroso & 1,0 & 1,0 \\
\hline 10 & ...Por motivos de viajes (por ejemplo, negocios, situaciones y/o, paseos familiares) & 0.5 & 0.5 \\
\hline 11 & $\begin{array}{l}\text {...Por falta de tiempo (por ejemplo: yo estoy muy ocupado, y/o tengo inconvenientes con los horarios de } \\
\text { las sesiones) }\end{array}$ & 1,0 & 1,0 \\
\hline 12 & ...Debido a las responsabilidades( por ejemplo del trabajo, estudio y tareas del hogar) & 0.8 & 0.8 \\
\hline 13 & ... Yo no tengo la energía (por ejemplo física y/o emocional) & 1,0 & 1,0 \\
\hline 14 & ...Debido a otros problemas de salud que me impiden ir a la rehabilitación (cuál:____ & 0.8 & 0.8 \\
\hline 15 & ...Yo me siento viejo & 0.5 & 0.5 \\
\hline 16 & ...Mi doctor no siente que sea necesario & 1,0 & 1,0 \\
\hline 17 & ...Muchas personas sufren del corazón y ellos no van a rehabilitación cardiaca y están bien & 1,0 & 1,0 \\
\hline 18 & ...Yo mismo puedo manejar mi problema del corazón y no necesito ayuda & 0,5 & 0.5 \\
\hline 19 & ...Yo pienso que fui remitido, pero el programa nunca me llamaron para programar una cita & 1,0 & 1,0 \\
\hline 20 & ...Paso mucho tiempo para ser remitido y entrar al programa de rehabilitación & 1,0 & 1,0 \\
\hline 21 & ...Yo prefiero cuidar mi salud solo y no en grupo & 0.8 & 0.8 \\
\hline \multirow[t]{2}{*}{22} & Tengo otro(s) motivo(s) para no asistir al programa de rehabilitación cardiaca:_ & 1,0 & 1,0 \\
\hline & IVC & 0,86 & 0,88 \\
\hline
\end{tabular}

R: Relevancia; P: Pertenencia; IVC: Índice de validez de contenido de la escala 
En la tabla 2 se observa la versión final de la escala, de donde los investigadores eliminan por consenso el ítem 18, teniendo en cuenta el IR y el IP, así como las sugerencias de los expertos. Es importante resaltar que la autora de la escala aprobó la eliminación de este ítem.

Tabla 2. Versión final Escala de Barreras para la Rehabilitación Cardiaca EBRC

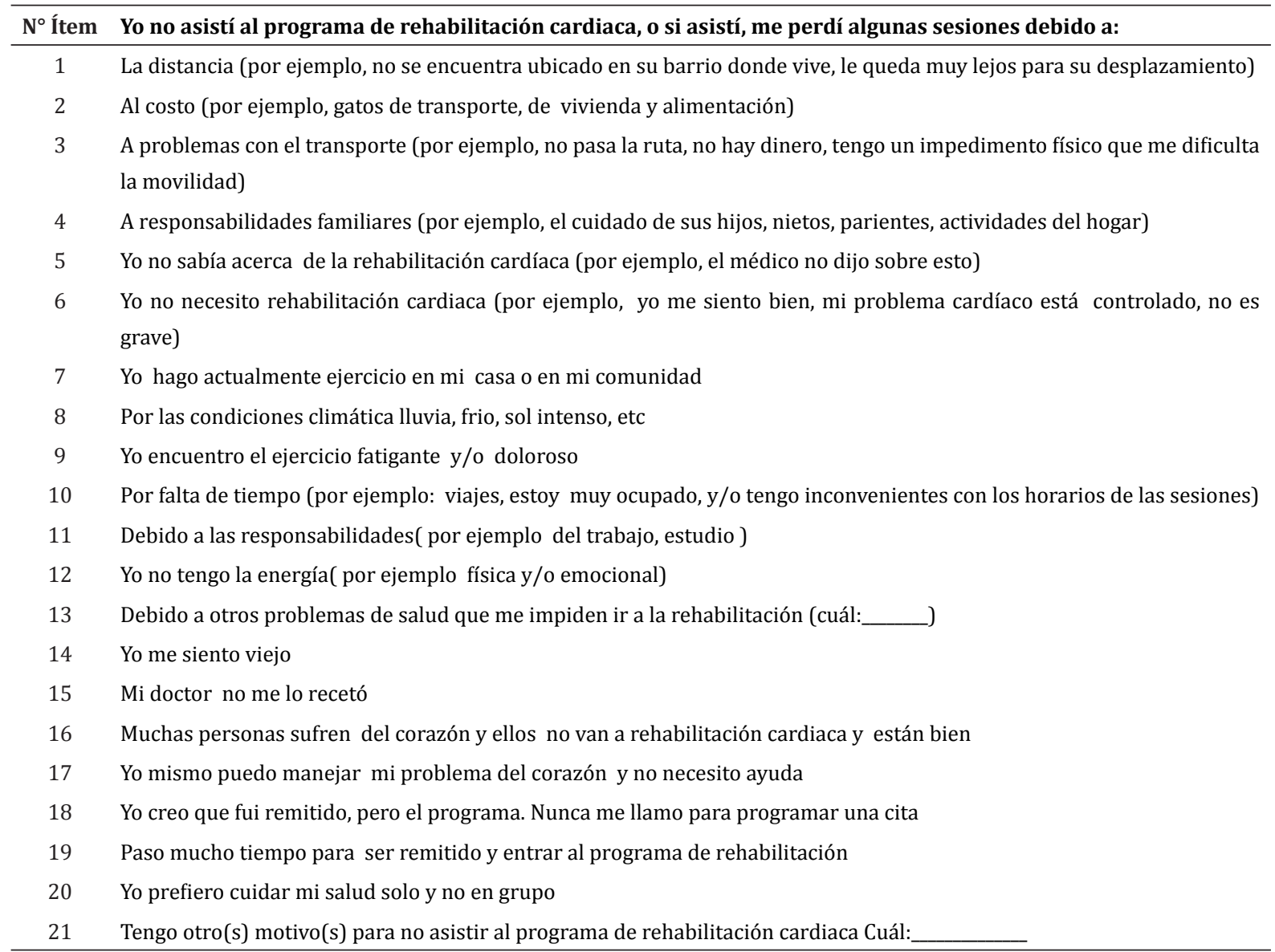

\section{Discusión}

Se realizó la adaptación transcultural de la EBRC teniendo en cuenta los pasos metodológicos sugeridos por García ${ }_{18}$ el cual se basa en las propuestas de

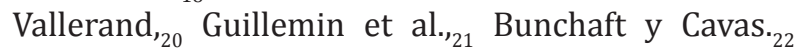
Por otra parte, se utilizó un panel de expertos para la determinación de la validez de contenido, tal como lo recomienda Polit et al., ${ }_{19}$ adicionalmente, se tuvo en cuenta las sugerencias emitidas por Lynn, $_{23}$ el cual recomienda un panel conformado entre 3 a 10 expertos. A través de este panel, se espera alcanzar equivalencia semántica, idiomática, experiencial y conceptual entre la versión local y la original; lo que frecuentemente demanda revisar y mejorar la redacción de los ítems del instrumento evaluado, haciendo caso a lo recomendado por Lepos et al $_{24}$

En el proceso de validación de contenido de la EBRC en población colombiana fue eliminado un ítem; no obstante, en el proceso de validación de la escala en versión portuguesa el número de ítems quedó igual a la escala original. Al respecto, es importante aclarar que en la versión portuguesa se realizó el proceso de 
adaptación cultural pero no se realizó la evaluación de la validez de contenido, sino que se procedió a realizar la evaluación de la validez de constructo. ${ }_{13}$

Omitir el proceso de validez de contenido podría considerarse un error puesto que, como mencionan Escobar-Pérez et al., ${ }_{25}$ "la sola adaptación del instrumento no necesariamente genera una equivalencia cultural debido a las barreras del idioma, a significados culturales diferentes de un constructo particular o a una variedad de interpretaciones de un comportamiento observado basado en normas culturales".

Dado que se contó con un panel de expertos, se obtuvo un concepto de personas con experticia en la materia, que además podían dar apreciaciones, observaciones o sugerencias. Aunque se realizaron algunas observaciones no se agregaron más ítems a los propuestos; se deja a juicio de los investigadores la posibilidad de incluir otros ítems, como aquellos relacionados con las barreras de acceso al Sistema de Seguridad Social en Salud existente en Colombia o incluir dentro de las investigaciones sobre barreras el tipo de seguridad social en salud.

Para finalizar, es importante resaltar que no hay escalas disponibles para evaluar barreras y accesibilidad a los servicios de rehabilitación cardiaca en el país, por lo cual, este trabajo se convierte en el primer paso en la búsqueda de los impedimentos más relevantes que disminuyen la participación y la adherencia a estos programas de prevención secundaria. Este trabajo hace parte de un proceso que viene adelantando el equipo de investigación CLINIUDES para describir las propiedades psicométricas, específicamente la confiabilidad de la escala aplicada a pacientes sometidos a angioplastia coronaria.

\section{Conclusiones}

Después de someter la escala al panel de expertos, se observó que su IVC-E es aceptable. Por otra parte, se hizo una revisión de los ítems 10, 15 y 18 los cuales tienen un IVC no aceptables $\leq 0,8$, y se realizaron las modificaciones pertinentes de acuerdo con las observaciones de los expertos, entre ellas la eliminación del ítem 18. Posteriormente se pretende completar el proceso de validación que incluye validez facial, de constructo, de criterio y la confiabilidad de la EBRC, para que finalmente pueda justificarse su utilización en el contexto colombiano.

Agradecimientos: Los autores agradecen a la Facultad de Salud y al programa de Fisioterapia de la Universidad de Santander UDES, por su apoyo y acompañamiento en la realización de la presente investigación.

Financiación: Esta investigación fue realizada con recursos de la Universidad de Santander UDES. Proyecto de convocatoria 017-13.

\section{Referencias}

1. Beltrán J, García M, Beltrán R, et al. Guías colombianas de cardiología - síndrome coronario agudo sin elevación del segmento ST. Rev Col Cardiol. 2008;15 (Supl 3):143-232.

2. Gallo J, Saldarriaga J, Clavijo M, Arango E, Rodríguez N, Osorio J. Actividad física y salud cardiovascular: En búsqueda de la relación dosis - respuesta. 1. Ed. Medellín: CIB; 2010.

3. Lavie C, Thomas R, Squires R, Allison G, Millani R. Exercise Training and Cardiac Rehabilitation in Primary and Secundary Prevention of Coronary Heart Disease. Mayo Clin Proc. 2009; 84(4): 373-383.

4. Shanmugasegaram S, Gagliese L, Oh P, Stewart, Brister SJ, Chan V, Grace SL. Psychometric validation of the cardiac rehabilitation barriers scale. Clin Rehabil. 2012; 26(2): 152-64.

5. Scane K, Alter D, Oh Paul, Brooks D. Adherence to a cardiac rehabilitation home program model of care: a comparison to a wellestablished traditional on-site supervised program. Appl Physiol NutR Metab. 2012; 37:206-213.

6. Menezes R. Cardiac rehabilitation and exercise therapy in the elderly: Should we invest in the aged?. J Geriatr Cardiol. 2012; 9:68-7.

7. Ghisi G, Polyzotis P, Oh P, Pakosh m, Grace S. Physician factors affecting cardiac rehabilitation referral and patient enrollment: A systematic review. Clin Cardiol. 2013;36(6):323-35.

8. Martin A, Woods C. What sustains long-term adherence to structured physical activity after a cardiac event?. J Aging Phys Act. 2012;20(2):135-47. 
9. Scane K, Alter D, Oh P, Brooks D. Adherence to a cardiac rehabilitation home program model of care: a comparison to a well-established traditional onsite supervised program. Appl Physiol Nutr Metab, 2012;37(2):206-213.

10. Clark A, King-Shier K, Spaling M, Duncan A, Stone J, Jagal $S$, et al. Factors influencing participation in cardiac rehabilitation programmes after referral and initial attendance: qualitative systematic review and meta-synthesis. Clin Rehabil. 2013; 27(10):948-59.

11. Fernandez RS, Salamonson Y, Juergens C, Griffiths R, Davidson P. Development and preliminary testing of the Cardiac Rehabilitation Enrolment Obstacle (CREO) scale: implications for service development. Eur J Cardiovasc Nurs. 2008;7(2):96-102.

12. York University. Sherry L. Grace, Phd. [Online] Canadá (Toronto). [Citado el 11 de Junio de 2015] Disponible en: http://www.yorku.ca/sgrace/crbarriersscale/ pproperties.html

13. Ghisi G, Scheveitzer V, Zulianello dos Santos R, Barros A, Recchi T, Oh P, et al. Desenvolvimento e validação da versãoem português da escala de barreiras para reabilitação cardíaca. Arq Bras Cardiol. 2012; 98(4):344-52

14. Mair V, Breda A, Boquembuzo M, Nagem L. Avaliação da aderência ao programa de reabilitação cardíaca em um hospital particular geral. Evaluating compliance to a cardiac rehabilitation program in a private general hospital. Einstein. 2013;11(3):278-84.

15. York University. [Online] Canadá (Toronto). [Citado el 11 de Junio de 2015] Disponible en: http://www. yorku.ca/sgrace/crbarriersscale/documents/CRBS_ Spanish_version.pdf

16. Orozco L. Medición en salud. Diagnóstico y evaluación de resultados. Un manual crítico más allá de lo básico. Colombia: Editorial y Publicaciones UIS; 2010.

17. Hernández R, Fernández C, Baptista P. Metodología de la investigación. 5ta edición. México: McGraw Hill; 2010.

18. García M, Montoya M, Medina E, Reyes M, Valenzuela J. Adaptación cultural de instrumentos de medida. Desarrollo Cientif Enferm. 2007;15(8): 369-72.

19. Polit DF, Beck CT. The content validity index: are you sure you know what's being reported? critique and recommendations. Res Nurs Health.2006; 29(5): 489-97.

20. Vallerand R. Vers une méthodologie de validation trans-culturelle de questionnaires. psychologiques: implications pour la recherché en langue française. Can Psychol. 1989; 30(4): 662-80.
21. Guillemin F, Bombardier C, Beaton D. Cross-cultural adaptation of health-related quality of life measures: Literature review and proposed guidelines. J Clin. Epidemiol, 1993; 46(12): 1417-32.

22. Bunchaft G, Cavas CST. Sob medida: Um guia sobre a elaboração de medidas do comportamento e suas aplicações. São Paulo: Vetor; 2002.

23. Lynn MR. Determination and quantification of content validity. Nurs Res. 1986; 35(6): 382 - 5.

24. Lepos A, Campos P, Felli V, Coggon D. Translation, adaptation and validation of the "Cultural and psychosocial influences on disability (CUPID) questionnaire" for use in brazil. Rev Lat Am Enfermagem. 2010; 18(6): 1092-8.

25. Escobar-Pérez J, Cuervo-Martínez A. Validez de contenido y juicio de expertos: una aproximación a su utilización. Avan Medic. 2008; 6;27-36. 